\title{
The valuation of the apparent density of a filter on a bicolored background
}

\author{
SERGIO CESARE MASIN and GIORGIO GARDONIO \\ University of Padua, Padua, Italy
}

\begin{abstract}
When the transmittance of a filter on a bicolored background is varied, the lightness differences that vary are those between the filter and the background and that between the parts inside the filter. It is hypothesized that these are the differences of importance for the valuation of the apparent density of the transparent layer. The results of two experiments seem to show that the rated apparent density does not depend only on the lightness difference inside the filter, or only on the two lightness differences between the filter and the background. All three lightness differences would enter the valuation mechanism. An averaging model is proposed.
\end{abstract}

Consider two big squares, one gray and one white, in contact along one side so as to form a bicolored rectangle. If we look through a rectangular filter at the contour where the squares touch (the contour of the filter being included within the contour of the rectangle), a pattern such as that shown in the inset in Figure 1 may result. Current theories and models of transparency (Beck, Prazdny, \& Ivry, 1984; Masin, 1984; Metelli, 1982, 1983) seem to concur eventually in indicating that the degree of tansparency in the pattern is a function of differences between the reduction lightnesses $a, b, p$, and $q$ of parts $A, B, P$, and $Q .{ }^{1}$ However, the nature of this functional relation is still a matter of inquiry.

A reasonable hypothesis seems to be that the lightness differences of importance are those that vary when the density of the filter is varied. Thus, the greater the density of the filter, the greater is the lightness difference between the filter and the background and the smaller is the lightness difference between the parts inside the filter.

In the case of the pattern in the inset in Figure 1, there are two lightness differences, $|\mathrm{a}-\mathrm{p}|$ and $|\mathrm{b}-\mathrm{q}|$, between the filter and the bicolored background and one lightness difference, $|p-q|$, between the two halves of the filter. How are these differences used by the perceptual system? On the assumption that perceived transparency is a function of relative similarity of lightnesses (Beck, Prazdny, \& Ivry, 1984), and on the basis of the hypothesis now set forth, the following three possibilities arise.

One might argue that only the difference $|p-q|$ is used. If lightness differences and the apparent density, $\psi$, of the filter vary in the interval $[0, k]$, then this possibility may be stated in symbolic terms as follows:

$$
\psi=\mathbf{k}-|\mathrm{p}-\mathrm{q}|
$$

Model 1 implies that if $\psi$ and $|p-q|$ are valuated using

Correspondence may be directed to S. C. Masin at Istituto di Psicologia, Piazza Capitaniato 3, 35139 Padova, Italy. numbers in the range $[0, \mathrm{k}]$, then the valuation of $\psi$ must coincide with $k$ minus the valuation of $|p-q|$. That is, $\psi$ increases as $|p-q|$ decreases.

However, one might argue that only the differences $|a-q|$ and $|b-q|$ are important, and that the observer compromises between them. In symbols

$$
\psi=w|a-p|+(1-w)|b-q|,
$$

where $w$ is a weight coefficient expressing the compromise. Model 2 implies that the valuation of $\psi$ must fall in between those of $|a-p|$ and $|b-q|$. (The models $\psi=|\mathrm{a}-\mathrm{p}|$ and $\psi=|\mathrm{b}-\mathrm{q}|$ are obvious special instances of Model 2 when $w=1$ and $w=0$, respectively.)

Clearly, Models 1 and 2 are special instances of the more general model

$\psi=w_{1}|a-p|+w_{2}|b-q|+\left(1-w_{1}-w_{2}\right)(k-|p-q|)$,

in which it is assumed that all three lightness differences are used. The weights $w_{1}$ and $w_{2}$ express a compromise between the three differences.

The following experiment was undertaken in order to check these models, and consequently determine which differences contribute to the rated density of a filter on a bicolored background. Evidently, only in the case where both implications of Models 1 and 2 turn out to be false may it be conlcuded that $\psi$ depends on all three differences. However, it is important to note that when this is the case Model 3 would be corroborated but not shown to be true.

\section{EXPERIMENT 1}

\section{Method}

Observers. Thirty university students served as unpaid observers. They were asked to participate in the experiment as they entered the institute. 
Table 1

Reflectance for Surfaces $P$ and $Q$ (Figure 1), and Virtual Reflectance and Transmittance of the Episcotister Rotating at Fusion Speed Simulated in the Experimental Pattern

\begin{tabular}{ccccc}
\hline $\begin{array}{c}\text { Number of } \\
\text { Pattern }\end{array}$ & $\begin{array}{c}\text { Reflectance } \\
\text { for Surface } \\
\mathbf{P}\end{array}$ & $\begin{array}{c}\text { Reflectance } \\
\text { for Surface } \\
\mathrm{Q}\end{array}$ & $\begin{array}{c}\text { Virtual } \\
\text { Reflectance }\end{array}$ & $\begin{array}{c}\text { Virtual } \\
\text { Transmittance }\end{array}$ \\
\hline 1 & .16 & .13 & .13 & .04 \\
2 & .19 & .13 & .14 & .08 \\
3 & .23 & .11 & .13 & .16 \\
4 & .40 & .08 & .12 & .43 \\
5 & .52 & .06 & .13 & .62 \\
6 & .67 & .04 & .10 & .86 \\
7 & .67 & .40 & .62 & .36 \\
8 & .59 & .31 & .49 & .38 \\
9 & .52 & .26 & .39 & .35 \\
10 & .46 & .19 & .29 & .36 \\
11 & .40 & .13 & .19 & .36 \\
12 & .35 & .08 & .14 & .36 \\
13 & .31 & .04 & .04 & .38 \\
\hline
\end{tabular}

Note-The reflectances for surfaces $A$ and $B$ were .76 and .02 , respectively.

Stimuli. Patterns made of gray paper were used. The patterns, placed on the frontal-parallel plane and viewed from a distance of $1.55 \mathrm{~m}$, had the form indicated in Figure 1 (the lengths of the side of the squares for $\mathrm{A}$ and $\mathrm{B}$ and for $\mathrm{P}$ and $\mathrm{Q}$ were, respectively, 40 and $22 \mathrm{~mm}$ ). The patterns were stuck on white cardboard and displayed on a background of the same white (reflectance of about .9-.95). The illumination level was of $375 \mathrm{~lx}$.

For the gray paper, we used 19 pieces of paper supplied by the NCS Institute (Sweden). By choosing the suitable reflectances for surfaces $A, B, P$, and $Q$ (Figure 1), the same proximal stimulation as that produced by episcotisters rotating at fusion speed may be obtained. The possible combinations of gray papers satisfying this requirement are not abundant. Here, we used combinations, first used by Gyulai (1976), that allowed for the construction of 13 patterns. The reflectances for the background surfaces $A$ and $B$ were, respectively, .76 and .02 . Table 1 lists the reflectances used for surfaces $P$ and $Q$, and the virtual reflectances and transmittances of the simulated episcotisters revolving at fusion speed. ${ }^{2}$ As may be seen, the virtual reflectance was kept constant in patterns 1 to 6 , and the virtual transmittance was kept constant in patterns 7 to 13 .

Procedures. As an introduction, the observer was shown seven photographs of a filter on a black-and-white checked background. The filter in the photographs appeared to have different densities. The term "density" was defined by describing this property as varying from filter to filter, and pointing out that the greater the density, the less the visibility of the part of the background seen through the filter. After being assured that the observer freely agreed with this description, the experimenter gave the instructions.

The observer sat at a table provided with a chinrest to keep the eyes level with the stimulus. He/she was shown each of the 13 patterns, one at a time, in random order. The task was to rate the apparent density of the transparent surface using numbers in the range of 0-100, where 0 represented perfect transparency (as for example in a windowpane) and 100 , opacity. No information was given as to the range of apparent densities.

Subsequently, the 13 patterns were presented randomly three times more. Each time, the observer had to rate one of the following lightness differences: $|a-p|,|b-q|$, or $|p-q|$. There are six possible orders of estimation of the differences. Six groups of five observers were formed. Each group was assigned a different order of estimation of the differences. The rating had to be performed using the numbers in the range $0-100$, where 0 represented absence of any lightness difference and 100 the maximum difference such as that occurring between two adjacent black and white surfaces. No information was given as to the range of differences.

The observer had to call out the number within 5-10 sec, after the pattern was placed on the white background, both for the rating of color density and of lightness differences. A session lasted about $30 \mathrm{~min}$.

\section{Results}

The results for patterns 1 to 6 are depicted in Figure 1. The mean numerical rating of color density, $\psi$, the mean rating of the lightness differences $|a-p|$ and $|b-q|$, and the value obtained by subtracting the mean rating of $|p-q|$ from $100(100=k$ in Model 3$)$ are represented on the ordinate. These estimates were contrasted with the virtual transmittance (abscissa) of the episcotister simulated in the pattern. Each data point represents the arithmetic mean of 30 numerical estimates. The vertical bars indicate the standard error. As may be seen, the mean numerical estimate of $\psi$ falls in between those of $|a-p|$ and $100-|p-q|$ for all six patterns. Since $\psi$ does not fall in between $|a-p|$ and $|b-q|$, Models 1 and 2 should be rejected. Only Model 3 is corroborated.

Inspection of individual results for patterns 7 to 13 revealed response strategies different from those used, by the same observers, for patterns 1 to 6 . In the case of patterns 7 to 13 , some observers produced completely opposite numerical estimates of apparent density. That is,

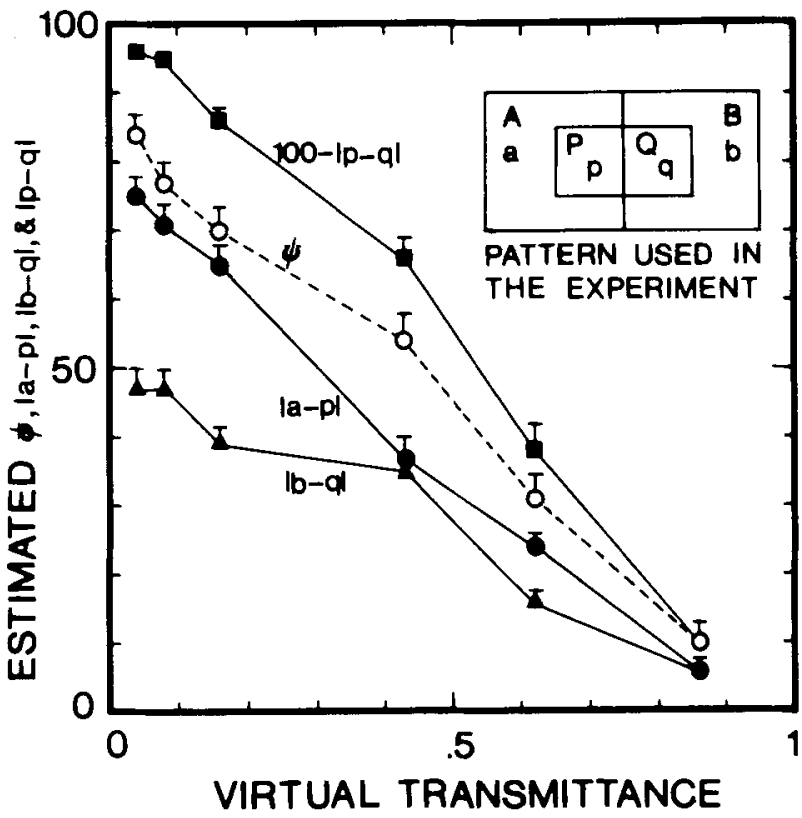

Figure 1. Results for patterns 1 to 6 (Table 1). Representation of the mean numerical estimates of color density, $\psi$, of the mean estimate of the lightness differences $|a-p|$ and $|\mathbf{b}-q|$, and of the value 100 minus the mean estimate of $|p-q|$, as a function of the virtual transmittance of the rotating episcotister simulated in the illustrated pattern. Each point is the arithmetic mean of $\mathbf{3 0}$ single estimates. The vertical bars indicate the standard error. Since $\psi$ is less than $100-|p-q|$, and is greater than both $|a-p|$ and $|b-q|$, it is concluded that all three lightness differences, $|\mathbf{p}-\mathbf{q}|,|\mathbf{a}-\mathbf{p}|$, and $|b-q|$, must enter the judgmental process. 
some of them arranged the patterns in order of increasing density, from 7 to 13 , and others ordered them from 13 to 7 . To select these observers impartially, the following criterion seemed appropriate. Let $E_{7}, E_{8}, \ldots, E_{13}$ be the numerical estimates of apparent density, respectively, for patterns $7,8, \ldots, 13$. If the estimates had the order relations $E_{7}>E_{11}, E_{8}>E_{12}$, and $E_{9}>E_{13}$, the observer was assigned to one group. If the relations were $E_{7}<E_{11}, E_{8}<E_{12}$, and $E_{9}<E_{13}$, the observer was as- signed to a second group. If neither of these order relations were met, the observer was assigned to a third group. The number of observers satisfying these criteria were, respectively, 7, 6, and 17 .

Figure 2 shows the results for these three groups and for the entire group of 30 . The arithmetic-mean estimates of $\psi$ produced by the two groups of 6 and 7 are represented as a function of the estimated lightness differences $|a-p|$ (Figure 2a) and $|b-q|$ (Figure 2b). The

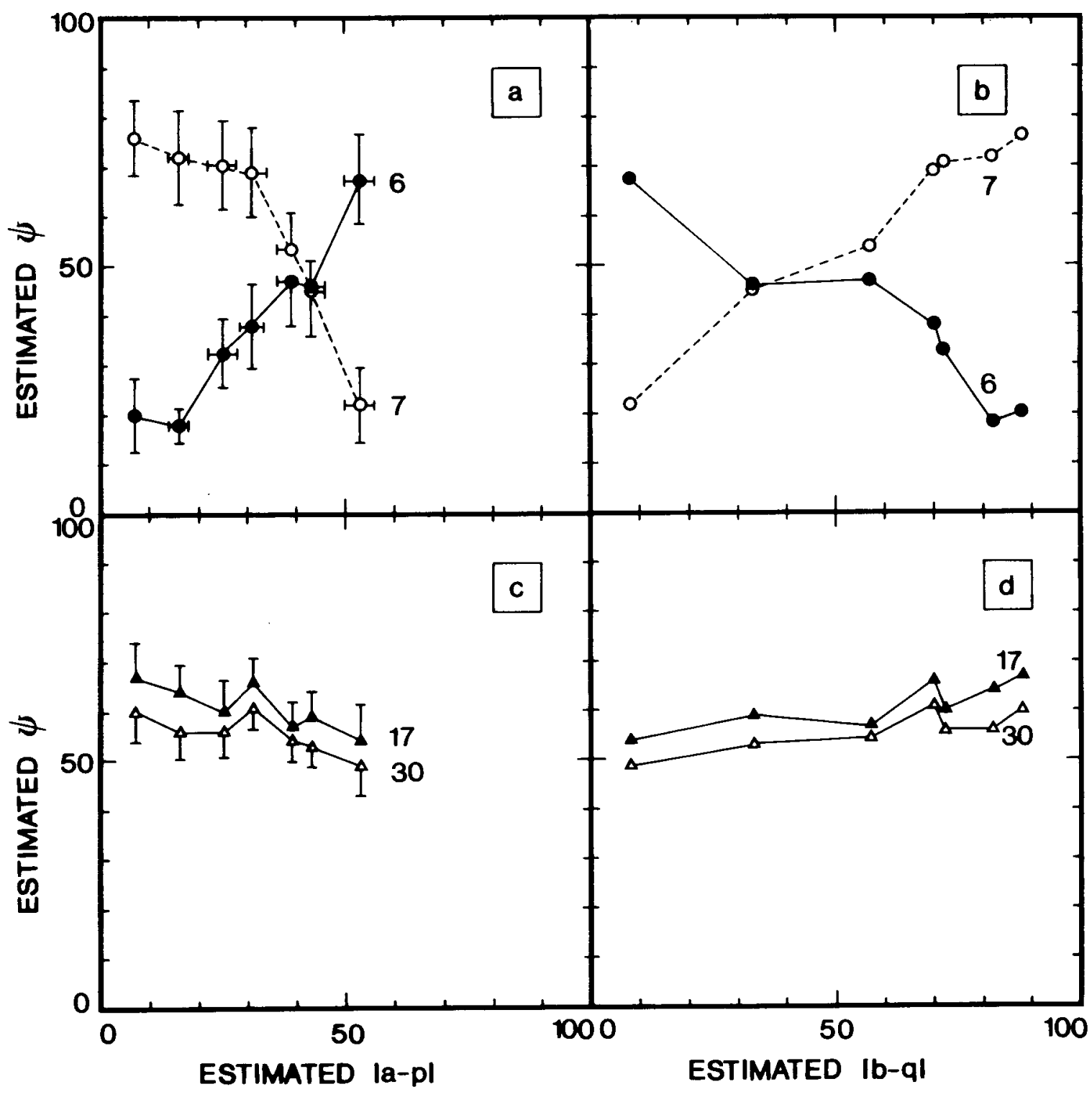

Figure 2. The 30 observers in Experiment 1 were subdivided into three subgroups of 6, 7, and 17, on the basis of a criterion described in the text. The mean estimate of color density, $\psi$, is represented as a function of the mean estimate of the lightness difference $|a-p|$ (diagrams a and $c$ ) and $|b-q|$ (diagrams $b$ and $d$ ) for each subgroup. The bars indicate the standard error of arithmetic means. The groups of 6 and 7 gave opposite estimates. This is due, plausibly, to the estimation of apparent density only in the region $P$, or $Q$, of the transparent surface. 


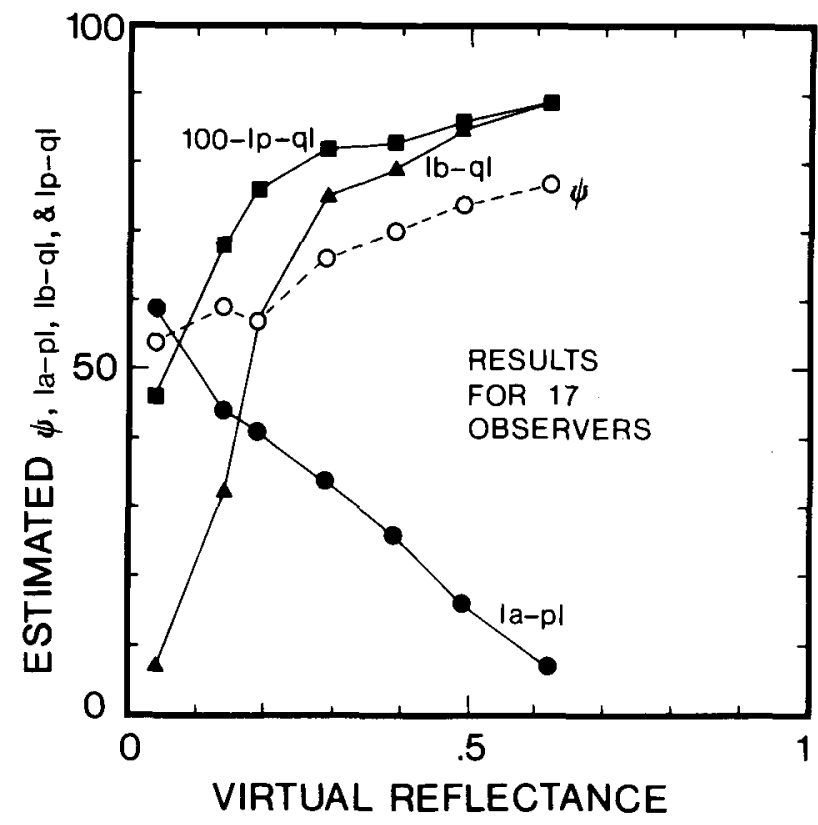

Figure 3. Results for patterns 7 to 13 (Table 1) for a group of 17 observers who could not be classified as were those in Figures $2 a$ and $2 \mathrm{~b}$. This figure should be interpreted in the same way as Figure 1. The substantial difference between the results shown in both this figure and Figure 2 and those shown in Figure 1 reveals that observers assumed a judgmental strategy that depended on the nature of the pattern. In experimental patterns 7 to 13, the color density in regions $P$ and $Q$ could be markedly different. The request to rate two perceptions (the densities in $P$ and in $Q$ ) by means of a single number obliges the observer to adopt a strategy different from that used when a single perception (equal density in $\mathbf{P}$ and $Q$ ) is rated.

standard errors are indicated only in Figures $2 \mathrm{a}$ and $2 \mathrm{c}$. As may be seen, the observers in the two groups of 6 and 7 gave quite opposite estimates for the same patterns. Gyulai (1976) obtained the same paradoxical results using the method of pair comparisons. She suggested that the results implied that these observers assumed an analytical attitude and valuated the density only in one of the two regions, $P$ or $Q$, of the transparent surface.

The reason why this particular strategy was used with patterns 7 to 13 , and not with patterns 1 to 6 , may be understood with reference to Figure 3 , which represents the results for the group of 17 observers. The mean $\psi,|a-p|$, $|b-q|$, and $100-|p-q|$ are represented on the ordinate. For purposes of repesentation, these estimates were contrasted with the virtual reflectance (abscissa) of the simulated episcotisters. The data points for $|\mathrm{a}-\mathrm{p}|,|\mathrm{b}-\mathrm{q}|$, and $100-|p-q|$ for the entire group of 30 almost superimpose those for the group of 17 represented in Figure 3. As may be seen, in Figure $1|a-p|$ and $|b-q|$ vary in the same direction, whereas in Figure 3 these two apparent differences vary in the opposite direction. This means that there were patterns (for example, 7 and 8 ) in which the difference $|a-p|$ was small and the difference $|b-q|$ was very large. In these cases, observers said they perceived a strong difference in density in the regions $P$ and $\mathrm{Q}$. Some said they had opted to rate only $\mathrm{P}$ and some that they had opted for $Q$. It is therefore plausible that, for this reason, 6 observers opted for $P$, and valuated $|a-p|$, and 7 opted for $Q$, and valuated $|b-q|$.

Some observers declared also that they had arrived at an average of the apparent density in $P$ and $Q$. The results for the 17 observers in Figure 3, who did not manifest a clear-cut preference for $P$ or for $Q$, might well have adopted that strategy. ${ }^{3}$ In fact, the estimates of $\psi$ (dashed line) lie in between those of $|a-p|$ and $|b-q|$. However, on the basis of these results, there is no way of telling to what extent, if any, $100-|p-q|$ contributed in the evaluation of apparent density.

\section{Discussion}

The results reported in Figure 1, which corroborate Model 3 and reject Models 1 and 2, should be reliable, because the results in Figure 2 agree with the previous findings of Gyulai (1976), who used a different psychophysical method. There is evidence (Curtis, 1970) that group category judgments of brightness differences are linearly related to differences between judgments of a single brightness. Due to the similarity between these two continua (Warren, 1969), the same conclusion should apply also to lightness. But this is not absolutely certain in the present stimulus conditions. Anderson (1976) and Weiss (1975) showed that the rating of lightness differences yields the same lightness scale as that obtained from the bisection task. However important these results may be, it is still possible that judgments of single lightness imply integration operations different from those occurring in judgments of lightness combinations (Anderson, 1981; Marks, 1974). It is therefore important to check whether the results reported in Figure 1 can be replicated when, instead of evaluating $|a-p|,|b-q|$, and $|p-q|$, the observer evaluates the reduction lightness $a, b, p$, and q (see Footnote 1).

\section{EXPERIMENT 2}

\section{Method}

Observers. Thirty other university students took part in this experiment as unpaid observers. They were recruited on entering the institute.

Stimuli. The stimuli were the patterns 1 to 6 used in Experiment 1 (Table 1). Observers placed their heads on a chinrest to keep their eyes level with the stimulus. The patterns were stuck in the middle of $10 \times 15 \mathrm{~cm}$ white pieces of cardboard, and displayed in the observer's frontal-parallel plane at a $1.7-\mathrm{m}$ distance. The illumination level was of about $25 \mathrm{~lx}$.

Procedure. The six patterns were presented in random order six times to each observer. Two subgroups, $X$ and $Y$, with 15 observers each, were formed. During presentations 1 and 2 of the entire series of six patterns, all 30 observers rated the color density of the transparent surface. During presentations 3 and 4, Subgroup $X$ rated the lightness of region $P$, and Subgroup $Y$ rated the lightness of $Q$. During presentations 5 and 6, Subgroup $Y$ rated the lightness of $P$ and Subgroup $X$ that of $Q$. Subgroup $X$ rated the lightness of region $A$ at the end of presentations 3 and 5 and the lightness 


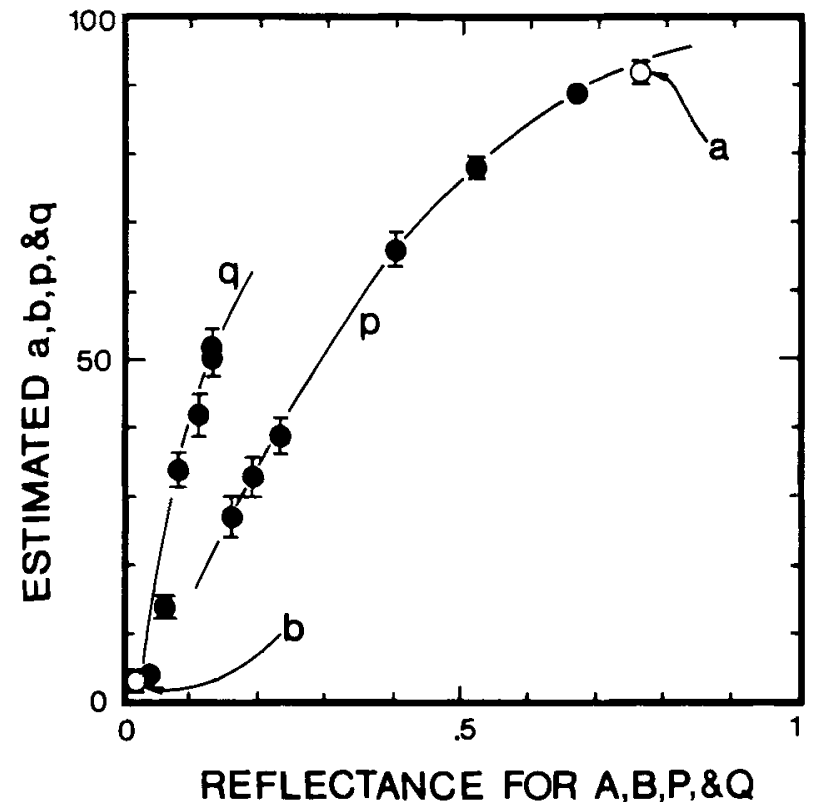

Figure 4. Psychophysical functions relating the mean rating of the reduction lightnesses $a, b, p$, and $q$ to the corresponding reflectances for surfaces A, B, P, and Q (patterns 1 to 6; Tahle 1). Filled dots represent the arithmetic mean of 30 single estimates of $p$ and $q$. The open dots represent the mean estimates of $a$ and $b$. The vertical bars indicate the double standard error.

of B at the end of presentations 4 and 6. For Subgroup Y, this order was reversed.

The instructions previously used in Experiment 1 were given for the estimation of color density. The instructions for the estimation of lightness were as follows. The observers were told to look at $P$ (or $Q$ ) as if it were a distinct surface separated from $Q(P)$. The observer had to rate, within 5-10 sec, the lightness of surfaces A, $B, P$, or $Q$, using numbers in the range of $0-100$. He/she was told that the number 0 represented the blackest black ever experienced in his/her life and 100, the whitest white. The number 50 represented the gray in between the blackest black and the whitest white. Other numbers represented intermediate grays. Observers were also allowed to use fractional numbers. A session lasted about $15 \mathrm{~min}$.

\section{Results}

The mean estimate of single reduction lightnesses $p$ and $q$ are represented as a function of the corresponding reflectance in Figure 4. The vertical bars show the standard error of the arithmetic means. The fact that the estimated $a$ and $b$ (unfilled dots) do not depart substantially from the corresponding curves confirms previous results of Masin and Quarta (1984).

From the mean estimates of $a, b, p$, and $q$, the differences $|a-p|,|b-q|$, and $100-|p-q|$ were computed for each pattern. These differences, together with the arithmetic mean of $\psi$, are represented on the ordinate in the diagram in Figure 5. The virtual transmittance is represented on the abscissa. The vertical bars indicate the standard error. Figure 5 should be compared with
Figure 1. As can be seen, $\psi$ falls in between $|\mathrm{a}-\mathrm{p}|$ and $100-|p-q|$ for patterns 2 to 6 in both figures.

\section{CONCLUSION}

The hypothesis set forth in the introduction was that the lightness differences of importance for the evaluation of apparent density of a transparent layer are those that vary when the transmittance of a filter, or a rotating episcotister, is varied. The results of the above experiments seem to show that the rated density does not depend only on the lightness differences between the filter and the bicolored background, or only on the lightness difference between the two halves of the filter. The rated density seems to depend on all three lightness differences.

The weight coefficients, $w_{1}$ and $w_{2}$, in Model 3 indicate the relative importance of the three lightness differences in the generation of the numerical response. These weights seem to vary both with the observer and with the involved lightness differences (Masin, 1984). Particularly dramatic changes of $w_{1}$ and $w_{2}$ were observed in Experiment 1 with patterns 7 to 13 . In some of these patterns, color density was markedly unbalanced. That is, region $P$ appeared much less dense than $Q$, for example, in pattern 7 and much more dense than $Q$ in pattern 13. As a result, some observers adopted the evident strategy of basing their responses on only one lightness difference. It

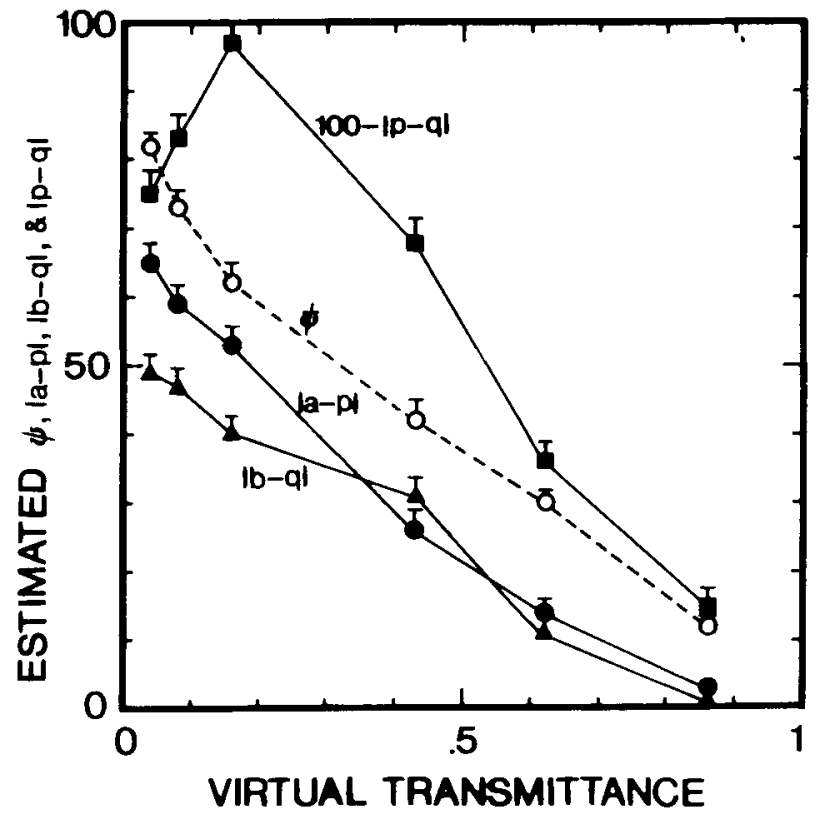

Figure 5. This figure should be interpreted in the same way as Figure 1. Instead of valuating the lightness differences $|a-p|$, $|\mathbf{b}-\mathbf{q}|$, and $|\mathbf{p}-\mathbf{q}|, 30$ observers valuated the single lightnesses $\mathbf{a}$, $b$, $p$, and $q$. The mean color differences represented as a function of the virtual tansmittance were obtained by taking the difference of the mean estimates of $a, b, p$, and $q$. The results represented in Figure 1 are substantially replicated. 
seems clear that without advance knowledge of the weight coefficients, only predictions at the ordinal level can be tested as in Experiments 1 and 2. Thus, Model 3 is corroborated, but not proved to be necessarily true. Some variant of it could imply the same ordinal predictions as well.

In looking at a transparency, as for example in Figure 1, we may adopt a normal or an analytical attitude. Under normal attitude, we perceive a single transparent surface, and do not heed lightness differences. Under the analytical attitude, we isolate the various regions in the field, and may heed lightness differences. Reasonably, the task of rating color density induces the observer to evaluate the degree of density in the various parts in the filter and, in so doing, triggers an analytical attitude that would consequently cause him or her to base the judgment on the lightness differences being attended to. It is also possible that the observer oscillates from an analytical to a normal attitude. Lightness differences would be heeded also in this case, although intermittently.

Perceptual models of transparency single out the lightness differences that the visual system would use (under the normal attitude) to generate the transparency phenomenon. Instead, Model 3 singles out the lightness differences that the valuation mechanism would use (reasonably, under the analytical attitude) to produce the judgment of color density. The determination of whether the observer assumes an analytical attitude or not, when evaluating color density, is a methodological problem which has, as yet, not been satisfactorily overcome. Therefore, a presently unresolved problem is the extent to which judgments approximate the target perceptual attribute under the normal attitude. In the case of judgments representing unbiasedly the degree of color density, Model 3, or similar versions of it, would compete with perceptual models as plausible descriptions of the operations underlying the generation of phenomenal tansparency.

\section{REFERENCES}

ANDERson, N. H. (1976). Integration theory, functional measurement, and the psychophysical law. In H.-G. Geissler \& Yu. M. Zabrodin (Eds.), Advances in psychophysics. Berlin: VEB Deutscher Verlag.

ANDERSON, N. H. (1981). Foundations of information integration theory. New York: Academic Press.

Beck, J., PrazdnY, K., \& IVRY, R. (1984). The perception of transparency with achromatic colors. Perception \& Psychophysics, 35, 407-422.
CURTIs, D. W. (1970). Magnitude estimations and category judgments of brightness and brightness interals: A two-stage interpretation. Journal of Experimental Psychology, 83, 201-208.

Ericsson, K. A., \& Simon, H. A. (1980). Verbal reports as data. Psychologial Review, 87, 215-251.

FuCHS, W. (1923). Experimentelle Untersuchungen über das simultane Hintereinandersehen auf derselben Sehrichtung. Zeitschrift für Psychologie, 91, 145-235.

GyulaI, E. (1976). Grado, colore e uniformità della trasparenza fenomenica. Atti dell'Istituto Veneto di Scienze, Lettere ed Arti, 134, 791-803.

Marks, L. E. (1974). Sensory processes, New York: Academic Press.

Masin, S. C. (1984). An experimental comparison of three-versus foursurface phenomenal transparency. Perception \& Psychophysics, 35, 325-332.

Masin, S. C., \& Quarta, A. (1984). Experimental demonstration that observers produce-unbiased estimates of reduction lightness in transparent surfaces. Bulletin of the Psychonomic Society, 22, 529-530.

METELLI, F. (1970). An algebraic development of the theory of perceptual transparency, Ergonomics, 13, 59-66.

METELLI, F. (1982). Stimulation and perception of transparency (Report No. 73). Padua, Italy: University of Padua, Institute of Psychology.

Metelli, F. (1983). Stimolazione e percezione della trasparenza con tonalità acromatiche. Ricerche di Psicologia, 26, 159-180.

NisBetT, R. E., \& Wilson, T. D. (1977). Telling more than we can know: Verbal reports on mental processes. Psychologial Review, 84, 231-279.

WARREN, R. M. (1969). Visual intensity judgments: An empirical rule and a theory. Psychological Review, 76, 16-30.

WEISs, D. J. (1975). Quantifying private events: A functional measurement analysis of equisection. Perception \& Psychophysics, 17, 351-357.

\section{NOTES}

1. The reduction lightness $p$, or $q$, is the gray color of part $P$, or $Q$, when transparency is not perceived. It was thought (Fuchs, 1923) that a strong analytical attitude could disrupt the perception of transparency in the filter. The reduction lightness of $P$, or $Q$, would then be the color perceived under an analytical attitude. Masin and Quarta (1984) have shown experimentally that the analytically seen color is, indeed, a reduction lightness.

2. Metelli (1970) gave equations that allowed the determinaton of the virtual transmittance of a simulated episcotister rotating at fusion speed, Let $m, n, x$, and $y$ be the reflectance for surfaces $A, B, P$, and $Q$. The virtual transmittance is given by $(x-y) /(m-n)$, and the virtual reflectance by $(m y-n x) /(m-n-x+y)$.

3. It should be recognized (Ericsson \& Simon, 1980; Nisbett \& Wilson, 1977) that observers may adopt these strategies even if they are unaware of them or if they are not able to verbalize, or remember, them. On the other hand, it is possible that when they do verbalize them, they do so only for a given pattern and later use a different strategy.

(Manuscript received December 11, 1984; accepted for publication December 11, 1984.) 